\title{
Lymph Node of Inguinal Region or Leg
}

National Cancer Institute

\section{Source}

National Cancer Institute. Lymph Node of Inguinal Region or Leg. NCI Thesaurus. Code C12362.

Any lymph node that is located in the inguinal region or in either leg. 